\title{
Neutrophils in primary gastric tumors are correlated with neutrophil infiltration in tumor-draining lymph nodes and the systemic inflammatory response
}

\author{
Soichiro Hiramatsu, Hiroaki Tanaka*, Junya Nishimura, Chie Sakimura, Tatsuro Tamura, Takahiro Toyokawa, \\ Kazuya Muguruma, Masakazu Yashiro, Kosei Hirakawa and Masaichi Ohira
}

\begin{abstract}
Background: Tumor-Associated Neutrophils (TANs) may be able to induce lymphangiogenesis and angiogenesis, although the detailed roles of TANs remain unclear. The Neutrophil-Lymphocyte Ratio (NLR) is an inflammation-based prognostic factor for gastric cancer. This study aimed to investigate the distribution of CD15 ${ }^{+}$neutrophils in the primary tumor and Tumor-Draining Lymph Nodes (TDLNs), and to examine the association of TANs with the clinicopathological features (including NLR) of patients with gastric cancer.

Results: Immunohistochemical staining showed that the median number of CD15 ${ }^{+}$TANs was 18 and 24 per highpower field (HPF) in primary tumors and TDLNs, respectively. Patients were divided into high and low infiltration groups based on the median number. A high number of infiltrating CD15 ${ }^{+}$TANs in the primary tumors and in the TDLNs were associated with depth of invasion and lymph node metastasis. Kaplan-Meier analysis revealed that a poor overall survival was associated with high numbers of CD15 TANs, and the multivariate analyses revealed that a high number of CD15 ${ }^{+}$TANs in the TDLNs was an independent prognostic factor. The numbers of CD15 1 TANs in the primary tumors and TDLNs showed weak positive correlation. The number of CD15 $5^{+}$ANs in the primary tumors was positively correlated with the preoperative $\mathrm{NLR},(P=0.001, R=0.327)$ and immunohistochemical staining revealed that C-X-C motif chemokine receptor 2 (CXCR2) ${ }^{+}$neutrophils might be the origin of the CD15 ${ }^{+}$TANs. Flow cytometry analysis indicated that infiltrating neutrophils increased in the tumor and TDLN compared to non-cancerous tissue. Neutrophils treated with cancer supernatant upregulated TWIST and IL-6 genes in vitro.
\end{abstract}

Conclusion: Our findings suggested that local infiltration of CD15 TANs may be correlated with inflammation in TDLNs and systemic response to cause metastasis in gastric carcinoma.

Keywords: Tumour-associated neutrophils, Gastric cancer, Prognosis, Draining lymph node

\section{Background}

Advanced gastric cancer has a poor prognosis, despite the development of novel treatments, and lymph node metastasis is an important prognostic factor in cases of gastric cancer $[1,2]$. We have previously reported that lymphangiogenesis was augmented in both of the primary gastric carcinoma and the tumour-draining lymph nodes (TDLNs) [3-5]. Furthermore, chronic inflammation involves

\footnotetext{
* Correspondence: hiroakitan@med.osaka-cu.ac.jp

Department of Surgical Oncology, Osaka City University Graduate School of Medicine, 1-4-3 Asahi-machi, Abeno-ku, Osaka 545-8585, Japan
}

immune cell infiltration, fibroblast proliferation, and angiogenesis, which are also observed in the tumor microenvironment [6, 7]. Moreover, intratumoral infiltration of immune cells is associated tumor invasion and metastasis [7-9]. Neutrophils are essential effector cells in the host's defence against invasive pathogens, and could promote tumor progression through angiogenesis, lymphangiogenesis, and immune suppression as Tumor-associated neutrophils (TANs) $[3,10-13]$. Although regional lymph nodes are considered an immune barrier for cancer, the detail mechanisms of lymph node metastasis remain

(c) The Author(s). 2018 Open Access This article is distributed under the terms of the Creative Commons Attribution 4.0 International License (http://creativecommons.org/licenses/by/4.0/), which permits unrestricted use, distribution, and 
unclear. Nevertheless, systemic inflammatory markers, including the Neutrophil-lymphocyte ratio (NLR), are prognostic factors in several cancers [13-15]. CD15, a carbohydrate adhesion molecule, is commonly used marker to identify human neutrophils and is known to mediate chemotaxis and phagocytosis. It has also been reported that some type of granulocytic myeloid-derived suppressor cells (MDSCs) which have immunosuppressive functions express CD15 [16]. Thus, CD15 is one of the effective molecules for searching the immunological function of neutrophils in the tumor microenvironment. Therefore, the present study aimed to explore the relationships of CD15 ${ }^{+}$TANs with gastric cancer and systemic inflammation, based on the microenvironments in the primary tumor and TDLNs.

\section{Methods \\ Patients and surgical specimens}

We retrospectively examined surgical specimens from patients who underwent gastrectomy at our department during 2007-2008. The specimens were formalin-fixed and paraffin-embedded tissues from 120 primary tumours and 497 TDLNs, which were analysed using immunohistochemistry. For the present study, TDLNs were defined as $4-5$ lymph nodes near the primary tumor. Pathological staging was performed according to the seventh edition of the

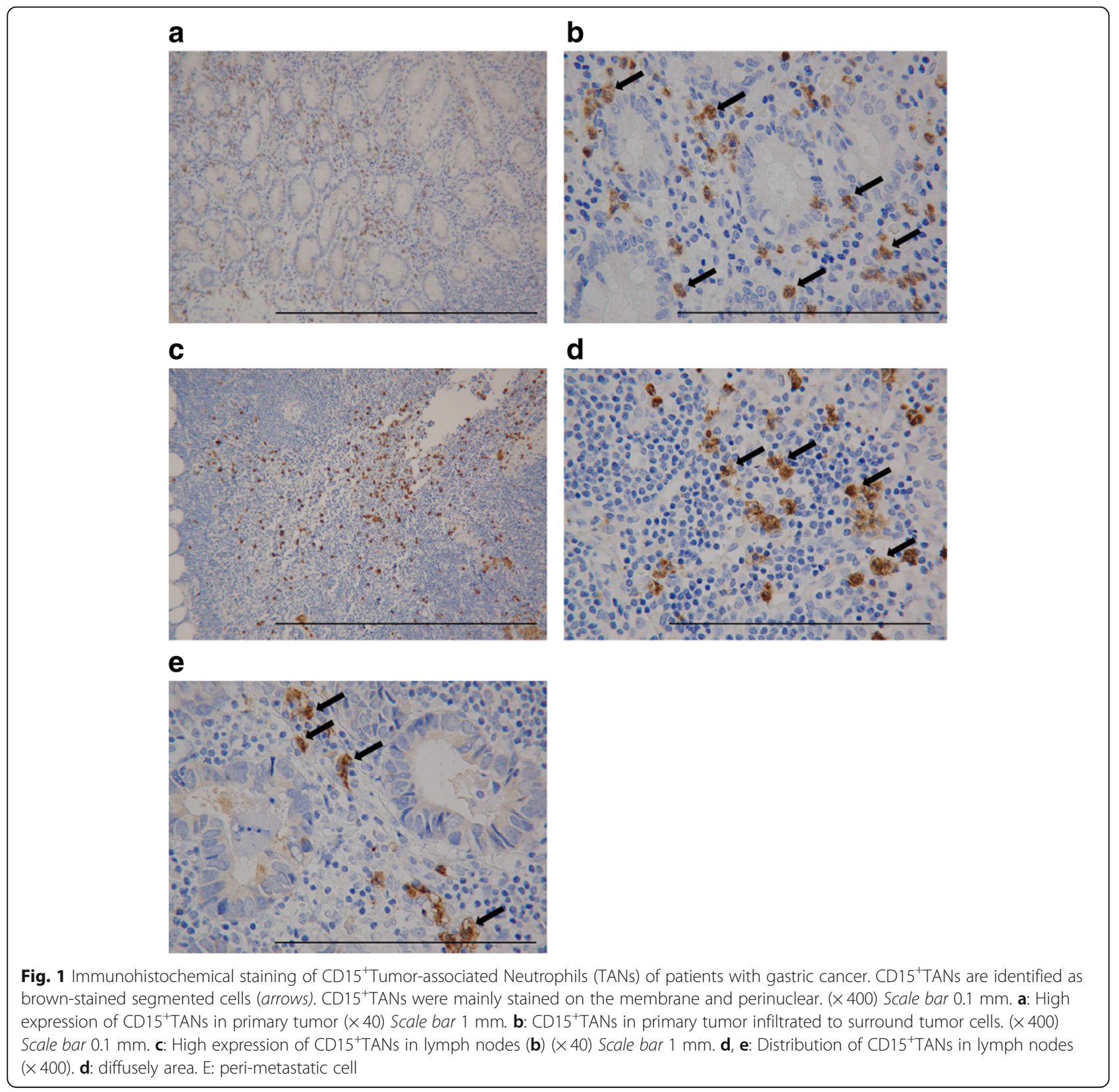


International Union Against Cancer Tumour-NodeMetastasis classification. Postoperative follow-ups were performed every 3 months for the first 2 years, and then every 6 months during years $3-5$.

\section{Immunohistochemical staining}

Sections with a thickness of $4 \mu \mathrm{m}$ were obtained from the paraffin-embedded blocks. After incubation at $60{ }^{\circ} \mathrm{C}$ for $10 \mathrm{~min}$, the sections were deparaffinized using xylene and rehydrated using a graded series of ethanol. The slides were subsequently washed twice for $5 \mathrm{~min}$ in phosphate-buffered saline (PBS). Endogenous peroxidase activity was blocked for 15 min using absolute methanol containing 3\% hydrogen peroxide. After washing the sections in PBS, they were microwaved for $10 \mathrm{~min}$ to achieve antigen retrieval. Nonspecific binding was blocked using a non-specific staining blocking reagent (Dako, Kyoto, Japan). The sections were then incubated overnight at $4{ }^{\circ} \mathrm{C}$ with mouse monoclonal antibodies (CD15 or C-X-C motif chemokine receptor 2

Table 1 Relationship between CD15 + TANs of primary tumour and clinicopathological features of patients with gastric cancer

\begin{tabular}{|c|c|c|c|c|}
\hline \multirow[t]{2}{*}{ characteristics } & \multirow[t]{2}{*}{$\mathrm{N}$} & \multicolumn{2}{|c|}{ TAN(primary tumour) } & \multirow[t]{2}{*}{$P$ value } \\
\hline & & high & low & \\
\hline \multicolumn{5}{|l|}{ Age(years) } \\
\hline$<65$ & 56 & 27 & 29 & \multirow[t]{2}{*}{0.987} \\
\hline$\geqq 65$ & 64 & 31 & 33 & \\
\hline \multicolumn{5}{|l|}{ gender } \\
\hline Male & 87 & 39 & 48 & \multirow[t]{2}{*}{0.023} \\
\hline female & 33 & 20 & 13 & \\
\hline \multicolumn{5}{|l|}{ pT category } \\
\hline $\mathrm{T} 1+\mathrm{T} 2$ & 69 & 24 & 45 & \multirow[t]{2}{*}{$<0.001$} \\
\hline $\mathrm{T} 3+\mathrm{T} 4$ & 51 & 34 & 17 & \\
\hline \multicolumn{5}{|l|}{$\mathrm{pN}$ category } \\
\hline$N(-)$ & 67 & 21 & 46 & \multirow[t]{2}{*}{$<0.001$} \\
\hline$N(+)$ & 53 & 38 & 15 & \\
\hline \multicolumn{5}{|l|}{ pStage } \\
\hline $\mid+\|$ & 78 & 27 & 51 & \multirow[t]{2}{*}{$<0.001$} \\
\hline$I I I+I V$ & 42 & 31 & 11 & \\
\hline \multicolumn{5}{|l|}{ Histology } \\
\hline differentiated(well, mod, pap) & 52 & 23 & 29 & \multirow[t]{2}{*}{0.1810} \\
\hline undifferentiated(por, sig, muc) & 68 & 36 & 32 & \\
\hline \multicolumn{5}{|l|}{ lymphatic invasion } \\
\hline absent & 48 & 15 & 33 & \multirow[t]{2}{*}{$<0.001$} \\
\hline present & 72 & 43 & 29 & \\
\hline \multicolumn{5}{|l|}{ venous invasion } \\
\hline absent & 91 & 39 & 52 & \multirow[t]{2}{*}{0.023} \\
\hline present & 29 & 10 & 19 & \\
\hline
\end{tabular}

$P$ value $<0.05$
(CXCR2), 1:100 dilution; Abcam, Tokyo, Japan), and subsequently washed using PBS for 10 min before a 10-min incubation with a secondary antibody at room temperature. After washing the sections using PBS, they were visualized using 3-3'-diaminobenzidine for $5 \mathrm{~min}$ and then counterstained using haematoxylin before mounting.

\section{Markers of systemic inflammation}

The NLR was retrospectively calculated based on routine test results from peripheral blood samples that were collected within 2 weeks before the operation. In cases with multiple blood samples, the sample from the first hospital visit was used to calculate the NLR. The median
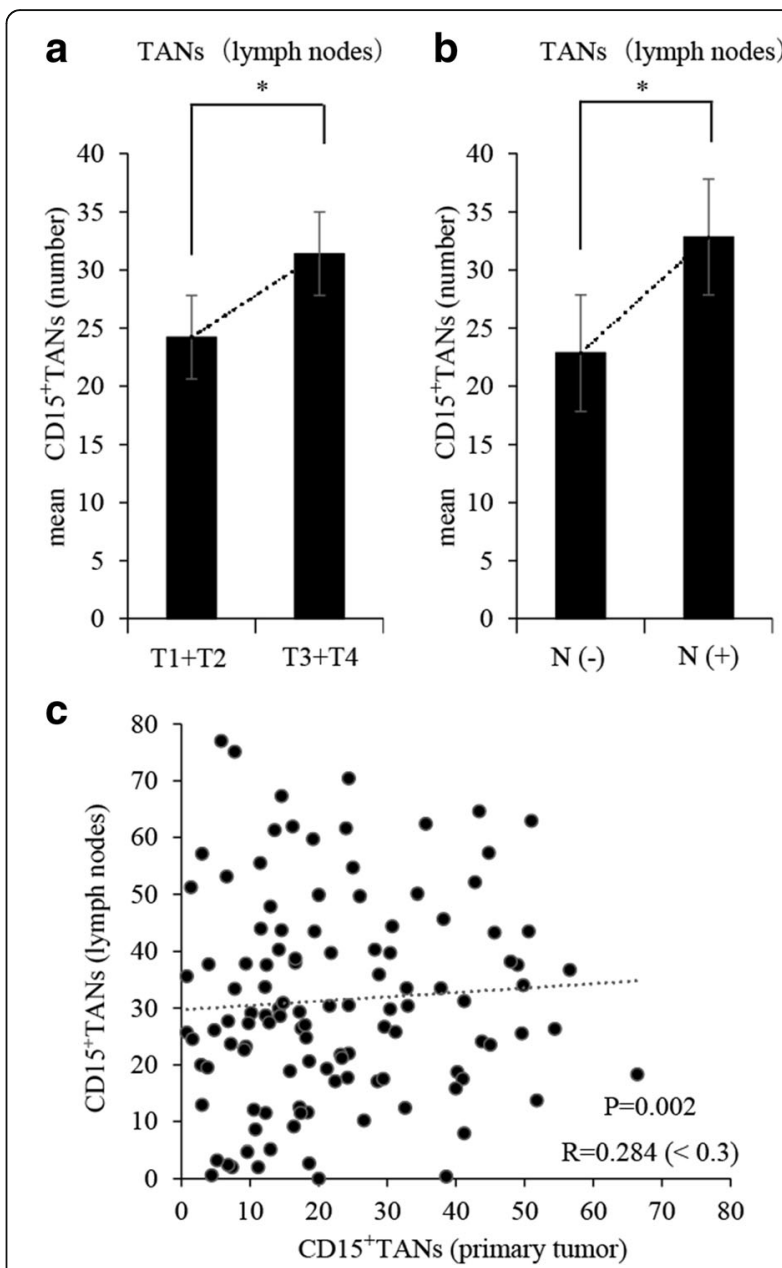

Fig. 2 Correlation between CD15 ${ }^{+}$TANs in lymph nodes and clinicopathological features of patients with gastric cancer, or CD15 ${ }^{+}$TANs in primary tumor. Based on the median number of CD15 ${ }^{+}$TAN in lymph nodes (23.9 cells/HPF), the patients were divided into high and low CD15 ${ }^{+}$TANs groups. $\mathbf{a}, \mathbf{b}$ : The number of CD15 $5^{+}$TANs in lymph nodes were significantly associated with pT3/4 disease $(P=0.036)$ and lymph node metastasis. $(P=0.002)$. $\mathbf{c}$ : A scatter chart of the number of TANs in primary tumor and in lymph nodes. Weak positive correlation was showed between TANs in primary tumor and in lymph nodes. $(P=0.002 R=0.284){ }^{*} p<0.05$ 
preoperative NLR value (1.932) was used as a cut-off to classify patients as having a high pre-operative NLR $(\geq 1$. 932) or a low pre-operative NLR $(<1.932)$. The numbers of TANs were compared between the high and low preoperative NLR groups.

\section{Flow cytometry}

Whole single-cell suspensions collected from tumor, nontumor region lymph nodes, and TDLNs were incubated with CD16b-PE antibodies (BD Pharmingen), and then analyzed by flow cytometry. Flow cytometric analyses were performed using a BD LSR II flow cytometer with FACSDiva $^{\mathrm{Tm}}$ software (both from Becton-Dickinson).

\section{Neutrophils isolation and culture}

Human neutrophils were isolated from healthy donors using Polymorphprep (Axis- Shield) centrifugation (purity $\geqq 85 \%)$. Cells were washed three times in complete RPMI 1640 (Sigma-Aldrich) (with $100 \mathrm{U} / \mathrm{ml}$ penicillin, $100 \mu \mathrm{g} / \mathrm{ml}$ streptomycin, and L-glutamine (Hyclone), and $10 \%$ fetal bovine serum (Gibco)). Then, neutrophils were incubated in complete RPMI 1640 at $37{ }^{\circ} \mathrm{C}$ and $5 \% \mathrm{CO} 2$ for $16 \mathrm{~h}$. OCUM12 were maintained in DMEM (Wako, Osaka, Japan) supplemented with 10\% fetal bovine serum (FBS; Nichirei Bioscience, Tokyo, Japan) and 20\% penicillin-streptomycin (Wako, Osaka, Japan). OCUM12 cell lines derived from human gastric cancer were suspended at a density of $1 \times 10^{5}$ per $\mathrm{ml}$ in RPMI1640 were incubated for $24 \mathrm{~h}$. Then we stimulated neutrophils from healthy donors using 50\% supernatant of OCUM12 for $24 \mathrm{~h}$. We defined neutrophils stimulated with the supernatant of OCUM12 as TAN in this experiment.

\section{Tumor-neutrophil co-culture system}

We seeded $1 \times 10^{5}$ OCUM12 cells in $500 \mu \mathrm{l}$ DMEM on 24-well plates, and neutrophils or TAN adjusted to $1 \times$ $10^{5}$ cells per $500 \mu \mathrm{l}$ DMEM were co-cultured with OCUM12 using cell culture Inserts (BD, Falcon) at $37^{\circ}$ $\mathrm{C}$ and 5\% CO2 for $16 \mathrm{~h}$. After $16 \mathrm{~h}$, OCUM12 was removed from medium, centrifuged, and washed three times by PBS. RNA was then extracted from the isolated OCUM12 cells.
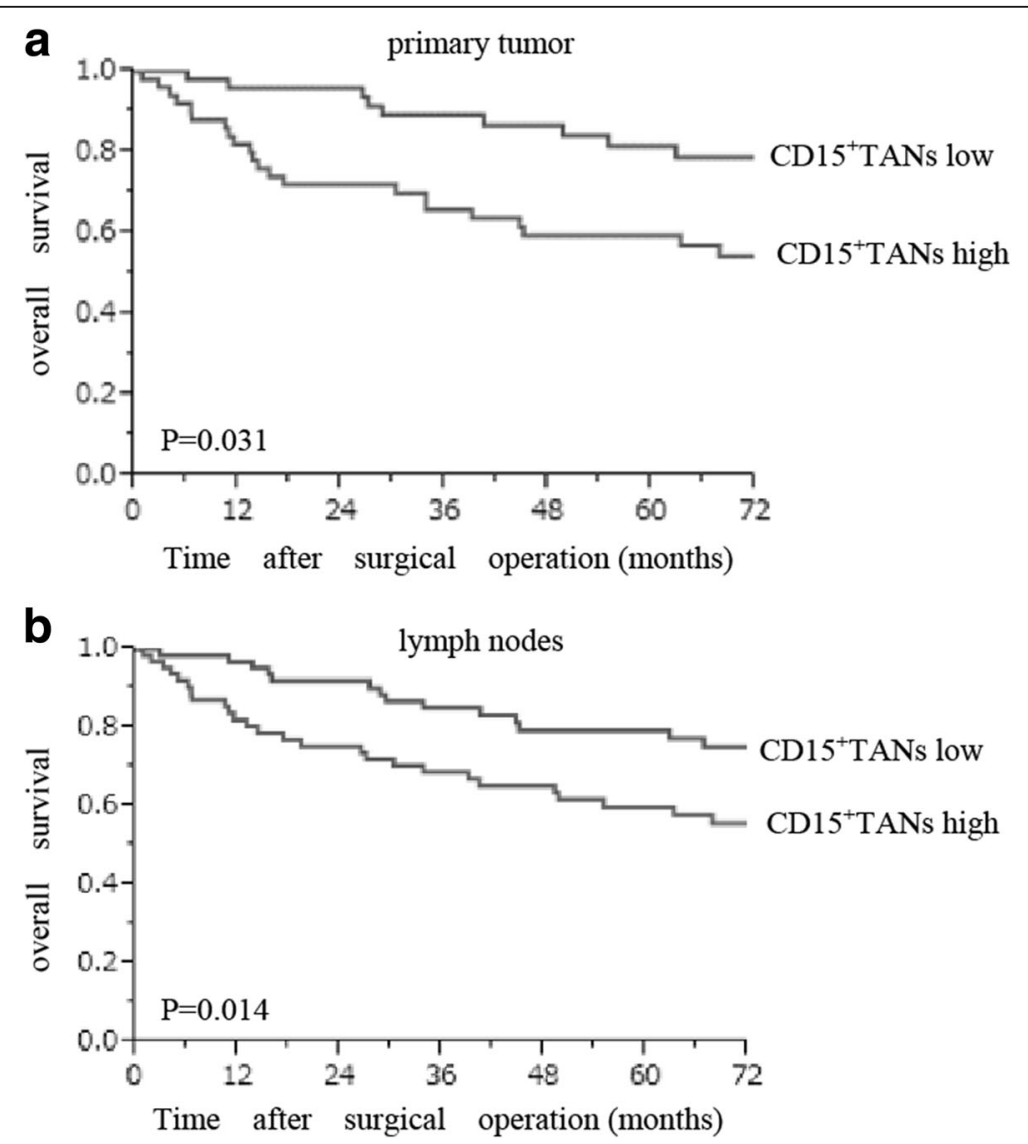

Fig. 3 The Kaplan-Meier survival analysis of CD15 ${ }^{+}$TANs in primary tumor and in lymph nodes. a: primary tumor b: lymph nodes. Based on the median number of CD15 TAN (primary tumors 18.4 cells/HPF lymph nodes 23.9 cells/HPF), the patients were divided into high and low CD15 ${ }^{\top}$ TANs groups. Log-rank test shows that both primary tumor and lymph nodes, patients with high CD15 ${ }^{+} T A N s$ group were poorer prognosis than those with low CD15 TANs group. (primary tumor: $P=0.031$, lymph nodes: $P=0.014$ ) 
RNA extraction and reverse transcription reactions

RNA easy Mini Kits (QIAGEN, Tokyo, Japan) were used according to the manufacturer's instructions to extract total RNA. ReverTra Ace qPCR RT Master Mix (TOYOBO, Osaka, Japan) was used according to the manufacturer's instructions to reverse transcribe the isolated RNA and generate single-strand cDNA; this reaction was performed at $37^{\circ} \mathrm{C}$ for $15 \mathrm{~min}, 50^{\circ} \mathrm{C}$ for $5 \mathrm{~min}$, and $98^{\circ} \mathrm{C}$ for $5 \mathrm{~min}$.

\section{Quantitative reverse-transcription PCR}

We analyzed the expression of mRNA of interleukin 6 (IL-6) in neutrophils. We also searched the expression of TWIST in OCUM12 via qRT-PCR. TaqMan PCR core reagents were used for these assays; each reaction was recorded and analysed with the ABI PRISM 7000 Sequence Detection System. After an initial denaturation for $10 \mathrm{~min}$ at $95^{\circ} \mathrm{C}$, each sample was subjected to 40 cycles of PCR $\left(95^{\circ} \mathrm{C}\right.$ for $15 \mathrm{~s}$, and $60^{\circ} \mathrm{C}$ for $1 \mathrm{~min}$, per cycle). To analyze the ratios of gene transcription levels, glyceraldehyde 3-phosphate dehydrogenase $(\mathrm{GAPDH})$ was used as an internal control. The $2^{-\Delta \Delta C t}$ method was used to determine the relative expression of each target gene. All experiments were performed in triplicate, and mean values were used for further calculation.

\section{Informed consent}

This study's retrospective protocol was approved by the Osaka City University ethics committee, and informed consent was obtained in writing from all patients and participants for collection and analysis of the specimens in this study.

\section{Statistical analysis}

Continuous variables were compared using Student's t test, and categorical variables were compared used the chi-square test. Overall survival curves were calculated using the Kaplan-Meier method and differences were evaluated using the log-rank test. Prognostic factors were examined using univariate and multivariate analyses with a Cox proportional regression model. Differences were considered statistically significant at $P$-values of $<0.05$. All statistical analyses were performed using JMP software (version 11; SAS Institute, Cary, NC, USA).

\section{Results}

Correlations of tumour-infiltrating CD15 ${ }^{+}$TANs with clinicopathological features

CD15 ${ }^{+}$TANs were identified based on brown membrane and perinuclear staining, and were observed to have infiltrated the primary tumour and surrounded the tumour cells (Fig. 1a, b). On the other hand, CD15 TANs in TDLNs were observed around metastatic cells (Fig. 1e), or diffusely areas (Fig. 1c, d).

Patients were divided into high and low TAN infiltration groups, based on median CD15 ${ }^{+} \mathrm{TAN}$ number of 18.4 per high-power field in primary tumours and 23.9 per high-powered field in lymph node.

High infiltration of $\mathrm{CD} 15^{+}$TANs in the primary tumor was associated with pT3/4 disease, lymph node metastasis, and positive vessel involvement (Table 1). Patients with

Table 2 Univariate and multivariate analysis of overall survival of patients with gastric cancer

\begin{tabular}{|c|c|c|c|c|}
\hline \multirow[t]{2}{*}{ factor } & Univariate analysis & $P$ value & Multivaliate analysis & $P$ value \\
\hline & \multicolumn{2}{|l|}{$\mathrm{HR}(95 \% \mathrm{Cl})$} & \multicolumn{2}{|l|}{$\mathrm{HR}(95 \% \mathrm{Cl})$} \\
\hline \multicolumn{5}{|l|}{ histology } \\
\hline undifferentiated vs differentiated & $1.805(1.138-2.933)$ & 0.011 & $2.155(1.084-4.540)$ & 0.028 \\
\hline \multicolumn{5}{|l|}{ pT Stage } \\
\hline$I I I+\mid V$ vs I + II & $5.546(3.334-9.633)$ & $<0.001$ & - & - \\
\hline \multicolumn{5}{|l|}{ pN Stage } \\
\hline$(+)$ VS $(-)$ & $4.129(2.500-7.092)$ & $<0.001$ & - & - \\
\hline \multicolumn{5}{|l|}{ pStage } \\
\hline$|I|+|V v s|+||$ & $5.124(3.193-8.355)$ & $<0.001$ & $3.116(1.330-7.977)$ & 0.008 \\
\hline \multicolumn{5}{|l|}{ lymphatic invasion } \\
\hline present vs absent & $3.882(2.228-7.251)$ & $<0.001$ & $0.594(0.151-2.218)$ & 0.443 \\
\hline \multicolumn{5}{|l|}{ venous invasion } \\
\hline present vs absent & $1.469(0.836-2.460)$ & 0.173 & - & - \\
\hline \multicolumn{5}{|l|}{ CD15 + TANs (primary) } \\
\hline high vs low & $2.157(1.081-4.588)$ & 0.028 & $1.391(0.676-3.038)$ & 0.376 \\
\hline \multicolumn{5}{|l|}{ CD15 + TANs (lymph) } \\
\hline high vs low & $2.150(1.122-4.278)$ & 0.013 & $1.997(1.036-4.051)$ & 0.038 \\
\hline
\end{tabular}

$H R$ Hazard ratio, $\mathrm{Cl}$ confidence interval. $P$ value $<0.05$ 
pT3/4 disease and lymph node metastasis had high numbers of $\mathrm{CD} 15^{+}$TANs (Fig. 2a, b). A scatter chart of $\mathrm{CD} 15^{+} \mathrm{TANs}$ in the primary tumor and TDLNs revealed a weak positive correlation $(P=0.002, r=0$. 284) (Fig. 2c). The Kaplan-Meier survival analysis of CD $15^{+}$TANs showed that both primary tumor and lymph nodes, patients with high $\mathrm{CD} 15^{+} \mathrm{TANs}$ group were poorer prognosis than those with low
CD15 ${ }^{+}$TANs group. (primary tumor: $P=0.031$, lymph node: $P=0.014$ ) (Fig. 3a, b). In addition, overall survival was univariately associated with depth of invasion, lymph node metastasis, International Union Against Cancer stage, histological type, lymphatic invasion, and a high number of $\mathrm{CD} 15^{+} \mathrm{TANs}$ in the primary tumour and in the TDLNs (Table 2). Multivariate analysis revealed that the independent

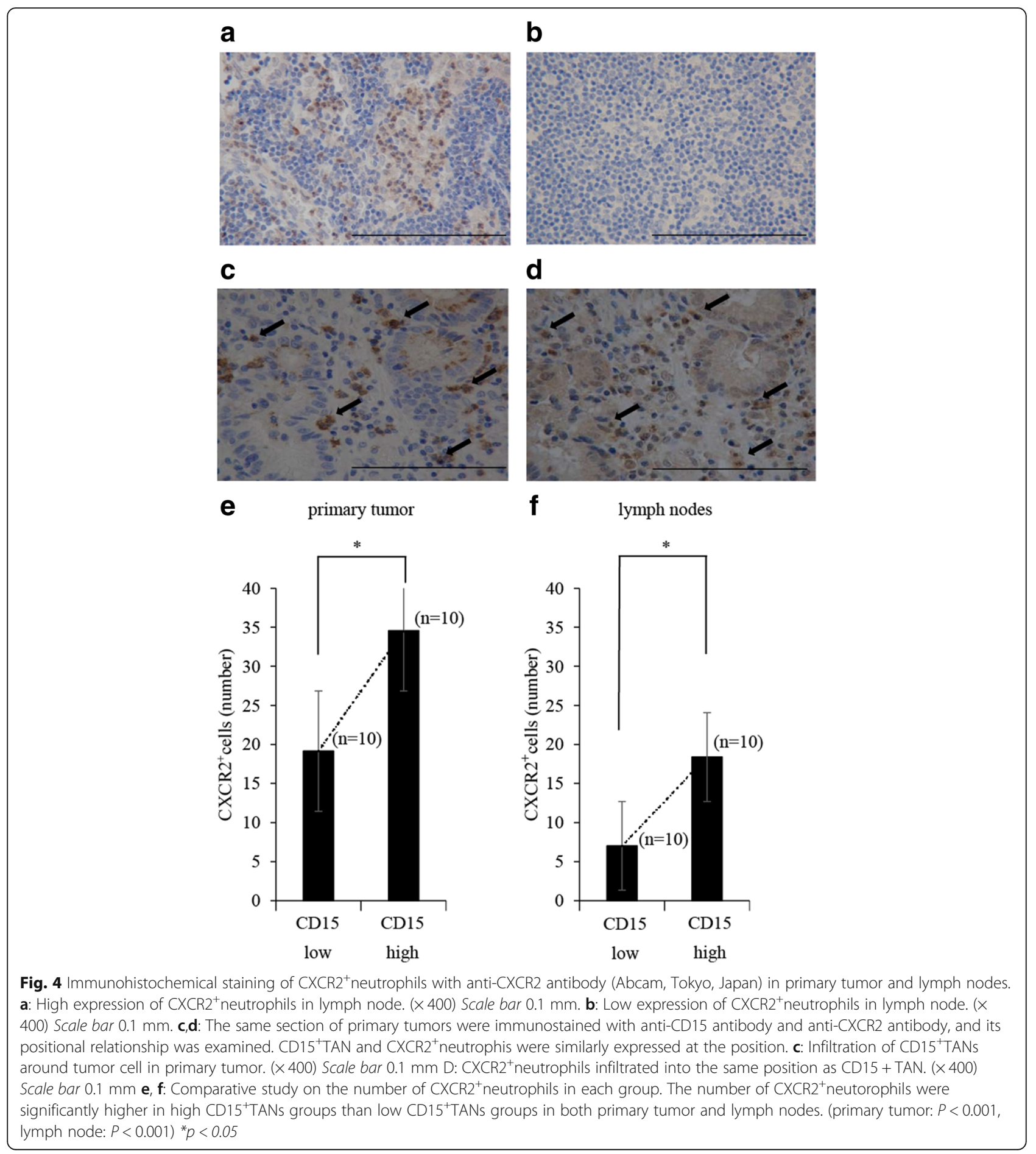


prognostic factors were undifferentiated type, a pathological stage of III/IV, and high $\mathrm{CD} 15^{+} \mathrm{TAN}$ infiltration in TDLN (Table 2).

\section{Expression of CXCR2 in the TANs}

We performed immunohistochemistry to detect CXCR2 expression to determine the origin of the TANs. CXCR2 ${ }^{+}$neutrophils were identified as brown-stained segmented cells that surrounded the tumour cells (Fig. 4d). We also observed that $\mathrm{CXCR}^{+}$neutrophils and $\mathrm{CD} 15^{+}$TANs exhibited similar infiltration locations in the same section of primary tumors (Fig. 4c, d). In both the primary tumors and the TDLNs, the expressions of CXCR2 were significantly elevated in the high $\mathrm{CD} 15^{+} \mathrm{TAN}$ group, compared to the low CD15 ${ }^{+} \mathrm{TAN}$ group (Fig. 4e, f).

\section{Correlation of TANs with the systemic inflammatory response}

We also explored the correlation of intratumoral TANs with the pre-operative NLR, as a marker of the systemic inflammatory response. A scatter chart of CD15 ${ }^{+}$TANs in the primary tumor and the pre-operative NLR revealed a positive correlation (Fig. $5 \mathrm{a}, \mathrm{P}=0.001, r=0$. 327). Furthermore, the average NLR value increased with tumor progression (Fig. $6 \mathrm{a}-\mathrm{c}$ ). The patients were subsequently divided into two groups based on the median pre-operative NLR value (1.93, range: $0-18.4$ ). Patients with a high NLR had a significantly higher average number of $\mathrm{CD} 15^{+}$TANs in the primary tumors, compared to patients with a low NLR (Fig. 6d).

\section{The effect of TANs on inflammation and metastasis}

Our results suggested that TAN may contribute to local inflammation and induction of metastasis. Therefore, we investigated a mechanistic link between TAN and inflammation or metastasis by in vitro experiment. Flow cytometry showed that the ratio of neutrophils in gastric cancer tended to be higher than that of normal mucosa. $(P=0.057)$ (Fig. 7a). In addition, neutrophils in metastatic lymph nodes were significantly more than lymph nodes without metastasis. $(P=0.016) \quad$ (Fig. 7b) To determine the effects of TANs on cancer cells, we also examined the expression of mRNA encoding EpithelialMesenchymal Transition (EMT) associated proteins (TWIST) in gastric cancer cell line, OCUM12. qRT-PCR showed that mRNA encoding TWIST were upregulated in OCUM12 co-cultured with TANs (3.32-fold). (Fig. 8a) Next, to examine the influence of TAN on inflammation, we compared the expression of mRNA coding IL-6 in neutrophils and TANs. The expression of mRNA encoding IL-6 in TANs were upregulated than that of neutrophils (1.48-fold) (Fig. 8b).
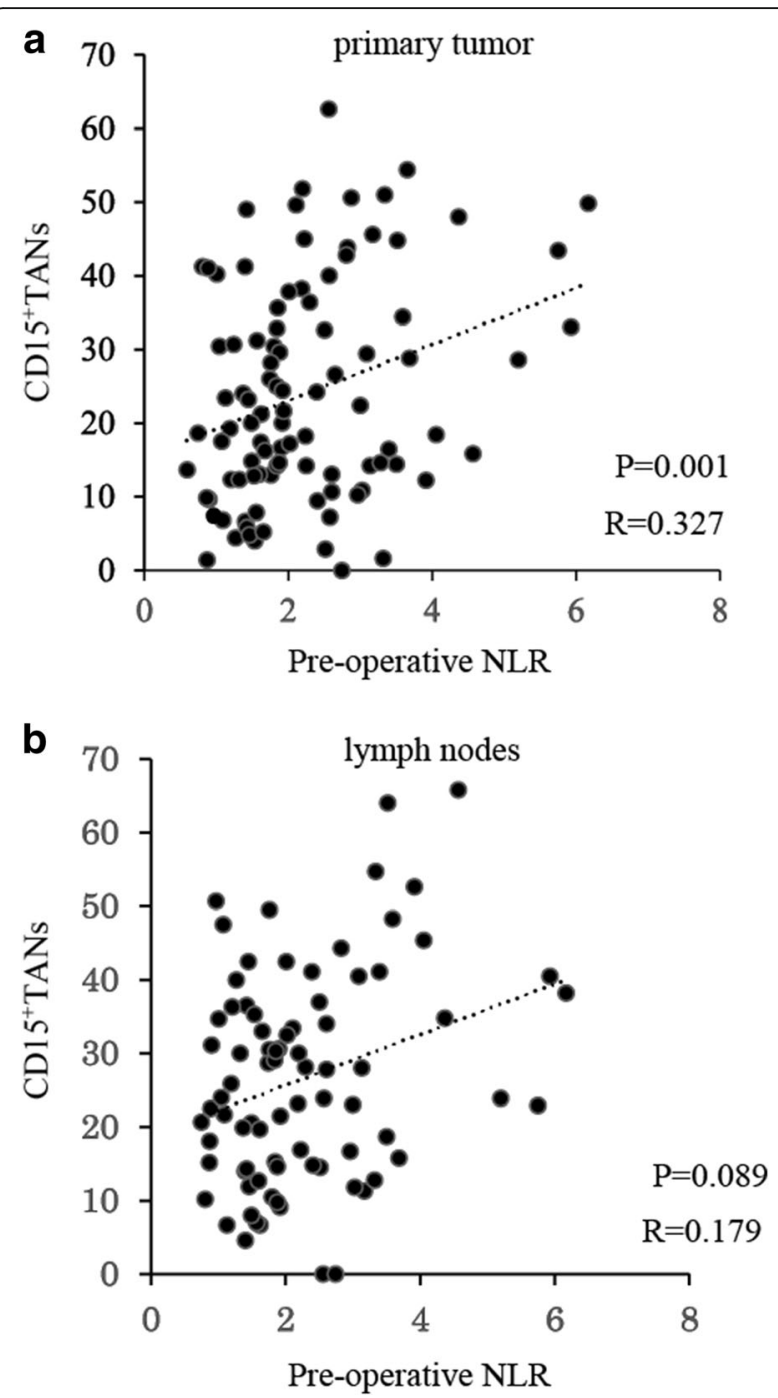

Fig. 5 a, b The scatter chart of CD15 TANs and pre-operative Neutrophil-to-Lymphocyte Ratio (NLR). a: In primary tumor, Positive correlation was observed between CD15 TANs and pre-operative NLR. $(P=0.001 R=0.327)$. b: There have no correlation between CD15 $15^{+}$ANs in lymph nodes and pre-operative NLR. $(P=0.089 R=0.179)$

\section{Discussion}

The present study revealed that the number of TANs in the primary tumors was associated with tumor progression and an increased number of TANs in the TDLNs. Furthermore, we observed that high TAN infiltration was correlated with the pre-operative NLR, which is a marker of systemic inflammation. CXCR2 ${ }^{+}$neutrophils and $\mathrm{CD} 15^{+}$TANs expressed similar distribution in the primary tumors. In addition, TANs had higher expression of IL-6 than normal neutrophils, and be increased the expression of TWIST which is known as one of the EMT marker. Our findings suggest that 

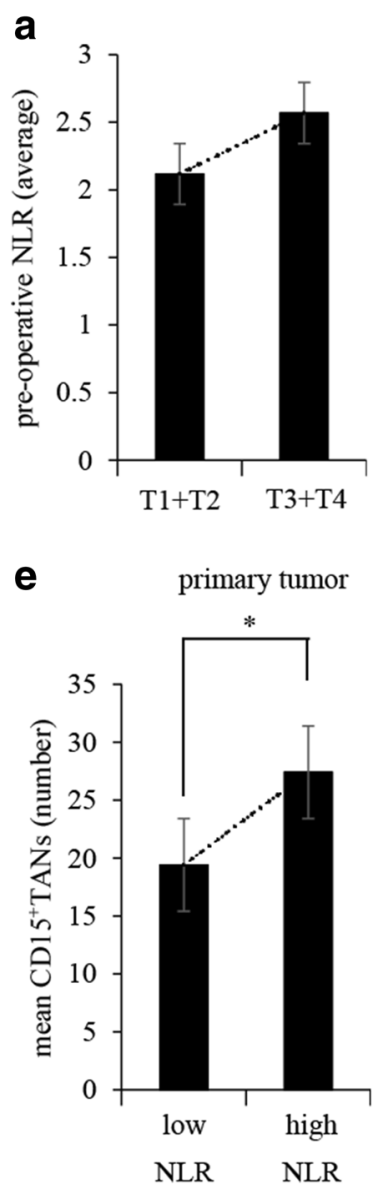

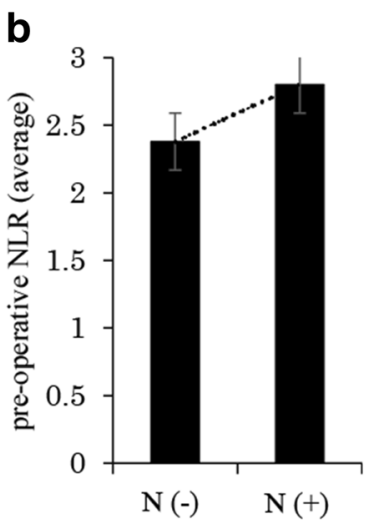

$\mathbf{f}$

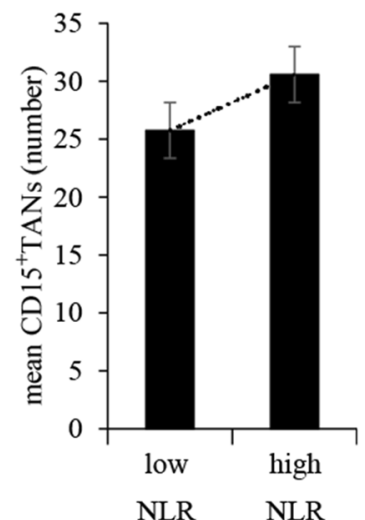

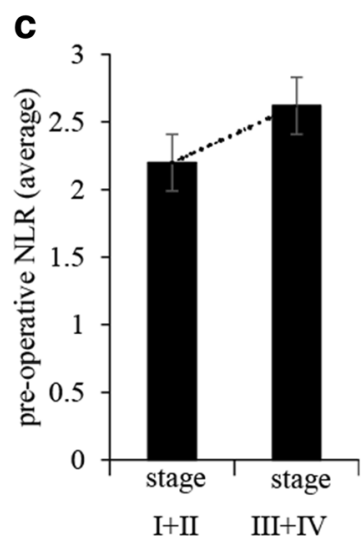

lymph nodes

Fig. 6 Correlation between pre-operative NLR of patients with gastric cancer and CD15 ${ }^{+} \mathrm{TANs}$ in gastric cancer tissue. a, b, c: Relationship between pre-operative NLR and tumor progression. $\mathbf{a}$ : pT3/T4 disease $\mathbf{b}$ : lymph nodes metastasis $\mathbf{c}$ : pStage. e, f: Comparative study on the number of CD15 TANs in each group. The patients were divided into two groups based on the median pre-operative NLR value. (1.93). The number of CD15 $5^{+}$ANs in primary tumors were significantly high in the high NLR group. $(P>0.001)$

tumor-infiltrating neutrophils might be related to metastasis of gastric cancer that is caused by inflammation.

Neutrophils are the most abundant white blood cells and reportedly contribute to tumour progression [17-19]. Moreover, neutrophils release several factors that can stimulate tumorigenesis and angiogenesis, such as vascular endothelial growth factor (VEGF), Matrix metalloproteinase9 (MMP9), Myeloperoxidase (MPO), cytokines, and chemokines [19, 20]. Neutrophils can also guide and shape the adaptive immune response, in which $\mathrm{T}$-cell proliferation is regulated by their production of several cytokines and growth factors [21]. T-cell suppression can also be mediated by extracellular arginase and reactive oxygen species, which are produced by neutrophils. Neutrophils also alter the inflammatory environment by producing L-arginine, arginase-1, and large amounts of reactive oxygen species. He et al. reported that peritumoral neutrophils up-regulate Programmed cell death ligand-1 (PDL-1) expression and suppress T-cell proliferation in hepatocellular carcinoma [10, 11], while Wang et al. have reported that tumor-derived Granulocyte macrophage colony-stimulating factor (GM-CSF) activates neutrophils and induces neutrophil PD-L1 expression through the Janus kinase and signal transducer and activator of transcription 3 (JAK-STAT3) pathway [11]. Other researchers have demonstrated that intratumoral neutrophils have prognostic value in cases of renal cell carcinoma, colorectal cancer, and non-small cell lung cancer [22-24], and we have reported that neutrophil infiltration is associated with lymph node micrometastasis and intranodal lymphangiogenesis [3]. In this study, we wanted to show the correlation of intratumoral neutrophils between primary tumor and TDLNs, and our results indicate that TANs in the primary tumor might spread through lymphatic vessels and become involved in cancer-related lymphangiogenesis.

Furthermore, we evaluated whether CD $15^{+}$TANs expressed CXCR2, which is a chemokine receptor that 


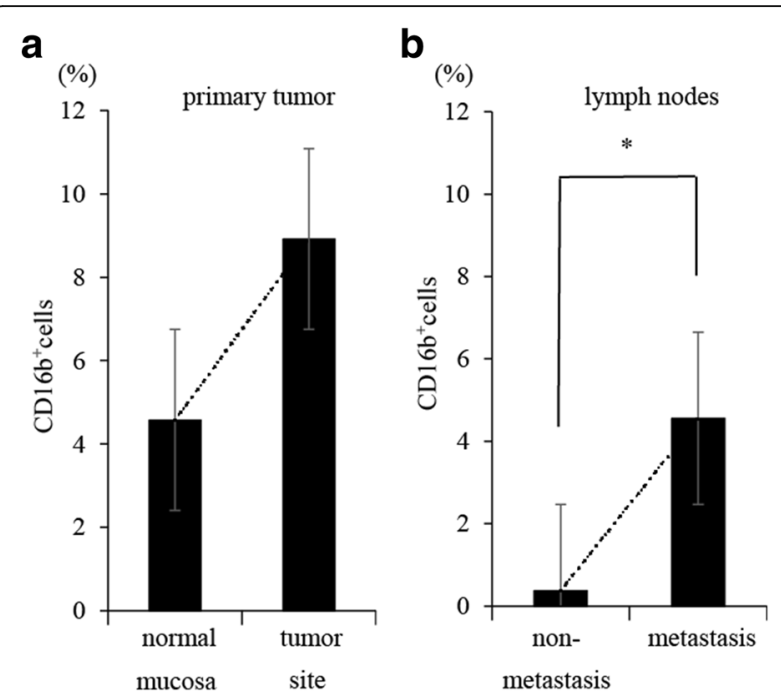

Fig. 7 Comparison of neutrophils infiltration between tumor area and normal area. Lymph nodes, primary tumors, and normal mucosa were collected from patients who underwent surgical resection for gastric cancer. We used these samples for making single cell suspensions, then analyzed the ratio of neutrophils in each samples by flow cytometry using anti-CD 16b antibody. (BD Biosciences, San Jose, CA, USA). a: Comparison between tumor area and normal mucosa. b: Comparison between metastasis lymph nodes and non-metastasis lymph nodes

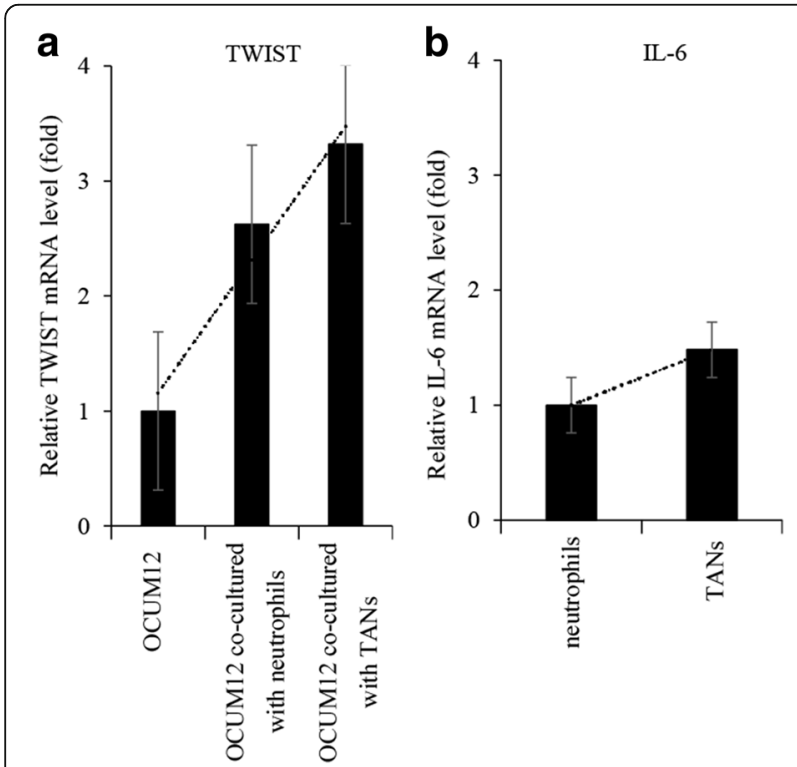

Fig. 8 Alteration in mRNA expression in OCUM12 co-cultured with TANs and induction of inflammation by TANs. We investigated the expression of TWIST in OCUM12 co-cultured with TANs by PCR arrays. We also compared the expression of mRNA coding IL-6 in TAN and normal neutrophils. We defined neutrophils stimulated with the $50 \%$ supernatant of OCUM12 as TAN in this experiment. a: TANs induced increases in expression of mRNAs encoding TWIST in OCUM12. $\mathbf{b}$ : Comparison of expression of IL-6 in TAN and normal neutrophils binds to C-X-C motif chemokine ligand (CXCL)1, CXCL3, CXCL5, and CXCL7. This receptor can regulate the recruitment and activation of neutrophils, and possibly mediate the inflammatory response in the tumor microenvironment [25, 26]. Previous studies have confirmed that CXCR2 ${ }^{+}$neutrophils are mainly observed in the stroma, where they play critical roles in mediating angiogenic activity, lymph node metastasis, and tumour proliferation in colon cancer, oral squamous cell cancer, oesophageal cancer, and breast cancer [26-29]. In addition, CXCR2+ neutrophils induce the migration of myeloid-derived suppressor cells into tumours [29]. Similarly, we observed that the numbers of CXCR2 ${ }^{+}$cells and TANs were correlated in the primary tumors, which indicates that TANs might have similar properties to those of myeloid-derived suppressor cells.

Many investigators have reported that the prognosis of patients with cancer can be predicted using systemic inflammatory indexes, including the NLR, the platelet-lymphocyte ratio, and the Glasgow prognostic score $[13,14,30,31]$. Among these indexes, NLR is believed to best reflect the host's immune status and the inflammatory response [13, 32]. The present study also revealed a positive correlation between the preoperative NLR and the number of CD $15^{+}$TANs in the primary tumor, and the average NLR value increased with tumor progression. These results suggest that excessive neutrophil infiltration into the tumor might mobilize neutrophils from the bone marrow into the peripheral blood, and that local inflammation caused by $\mathrm{CD} 15^{+} \mathrm{TANs}$ may be correlated with systemic inflammation in patients with gastric cancer.

The present study has several limitations. First, we only considered a small number of cases, and the cellular interactions between the tumor cells and CD $15^{+}$TANs were not directly observed using an experimental model. Thus, an in vitro experiment will be needed to evaluate the immune regulation that is performed by TANs. Second, we did not determine the neutrophils' differentiation cluster(s), such as CD66b, CD16b, CD32, and MPO [31, 33-36]. Third, we could not determine how CD $15^{+}$TANs cause tumor progression, induce lymph node metastasis, or spread throughout the body.

\section{Conclusion}

We found that patients with high $\mathrm{CD} 15^{+}$neutrophil infiltration in gastric cancer tissues had a poorer prognosis, compared to patients with low infiltration. In addition, the number of intratumoral neutrophils was associated with the systemic inflammatory response. Therefore, our findings suggest the TANs might play an important role in the induction of inflammation, which might allow gastric carcinoma cells to spread and induce lymph node metastasis. 


\section{Abbreviations}

CXCL: C-X-C motif chemokine ligand; CXCR2: C-X-C motif chemokine receptor 2; EMT: Epithelial-Mesenchymal Transition; FBS: Fetal bovine serum; GM-CSF: Granulocyte macrophage colony-stimulating factor; IL-6: interleukin 6; JAK-STAT3: Janus kinase and signal transducer and activator of transcription 3; MMP: Matrix metalloproteinase; MPO: Myeloperoxidase; NLR: Neutrophil-lymphocyte ratio; PBS: Phosphate-buffered saline; PDL1: Programmed cell death ligand-1; TANs: Tumor-associated neutrophils; TDLNs: Tumor-draining lymph nodes; VEGF: Vascular endothelial growth factor

\section{Funding}

Hiroaki Tanaka was supported by Grants-in-Aid for Scientific Research from the Ministry of Education, Culture, Sports, Science and Technology (grant no.26461990) for this study.

\section{Availability of data and materials}

The datasets used and/or analyzed during the current study are available from the corresponding author on reasonable request.

\section{Authors' contributions}

$\mathrm{SH}, \mathrm{JN}$, and CS performed experiments in the article. $\Pi$, $\Pi$, and $\mathrm{KM}$ contributed to collect samples, analysis, and data management. MY provided the materials and carried out additional experiments of revision with SH. HT contributed to interpretation of data and review of the article. $\mathrm{KH}$ and $\mathrm{MO}$ provided supervision of experiments. All authors read and approved the manuscript.

\section{Ethics approval and consent to participate}

All experimental procedures were approved as No. 3138 by the Osaka City University ethics committee, and all patients had provided informed consent for collection and analysis of the specimens.

\section{Competing interests}

The authors declare that they have no competing interests.

\section{Publisher's Note}

Springer Nature remains neutral with regard to jurisdictional claims in published maps and institutional affiliations.

\section{Received: 17 October 2017 Accepted: 4 April 2018 \\ Published online: 16 April 2018}

\section{References}

1. Ciliberto D, Staropoli N, Caglioti F, Gualtieri S, Fiorillo L, Chiellino S, De Angelis AM, Mendicino F, Botta C, Caraglia M, et al. A systematic review and meta-analysis of randomized trials on the role of targeted therapy in the management of advanced gastric cancer: evidence does not translate? Cancer Biol Ther. 2015;16(8):1148-59.

2. Zhao JJ, Pan K, Wang W, Chen JG, Wu YH, Lv L, Li JJ, Chen YB, Wang DD, Pan QZ, et al. The prognostic value of tumor-infiltrating neutrophils in gastric adenocarcinoma after resection. PLoS One. 2012;7(3):e33655.

3. Watanabe M, Tanaka H, Ohira M, Yoshii M, Sakurai K, Toyokawa T, Kubo N, Yamamoto A, Muguruma K, Yamashita Y, et al. Intranodal lymphangiogenesis precedes development of lymph node metastasis and accelerates progression of gastric cancer. J Gastrointest Surg. 2014; 18(3):481-90.

4. Tokumoto M, Tanaka H, Ohira M, Go Y, Okita Y, Sakurai K, Toyokawa T, Kubo N, Muguruma K, Maeda K, et al. A positive correlation between neutrophils in regional lymph nodes and progression of gastric cancer. Anticancer Res. 2014;34(12):7129-36.

5. Go Y, Tanaka H, Tokumoto M, Sakurai K, Toyokawa T, Kubo N, Muguruma K, Maeda K, Ohira M, Hirakawa K. Tumor-associated macrophages extend along lymphatic flow in the pre-metastatic lymph nodes of human gastric Cancer. Ann Surg Oncol. 2016;23(Suppl 2):S230-5.

6. Acharyya S, Massague J. Arresting supporters: targeting neutrophils in metastasis. Cell Res. 2016;26(3):273-4.

7. Coussens LM, Werb Z. Inflammation and cancer. Nature. 2002; 420(6917):860-7.

8. Smith HA, Kang Y. The metastasis-promoting roles of tumor-associated immune cells. J Mol Med (Berl). 2013;91(4):411-29.
9. Liu K, Yang K, Wu B, Chen H, Chen X, Chen X, Jiang L, Ye F, He D, Lu Z, et al. Tumor-infiltrating immune cells are associated with prognosis of gastric Cancer. Medicine (Baltimore). 2015;94(39):e1631.

10. Bowers NL, Helton ES, Huijbregts RP, Goepfert PA, Heath SL, Hel Z. Immune suppression by neutrophils in HIV-1 infection: role of PD-L1/PD-1 pathway. PLOS Pathog. 2014;10(3):e1003993.

11. Wang TT, Zhao YL, Peng LS, Chen N, Chen W, LV YP, Mao FY, Zhang JY, Cheng $P$, Teng YS, et al. Tumour-activated neutrophils in gastric cancer foster immune suppression and disease progression through GM-CSF-PD-L1 pathway. Gut. 2017;66:1900-11.

12. Uribe-Querol E, Rosales C. Neutrophils in Cancer: two sides of the same coin. J Immunol Res. 2015;2015:983698.

13. Yu L, Lv CY, Yuan AH, Chen W, Wu AW. Significance of the preoperative neutrophil-to-lymphocyte ratio in the prognosis of patients with gastric cancer. World J Gastroenterol. 2015:21(20):6280-6.

14. Duan H, Zhang X, Wang FX, Cai MY, Ma GW, Yang H, Fu JH, Tan ZH, Meng $Y Q, F u X Y$, et al. Prognostic role of neutrophil-lymphocyte ratio in operable esophageal squamous cell carcinoma. World J Gastroenterol. 2015;21(18):5591-7.

15. Tao L, Zhang L, Peng Y, Tao M, Li G, Xiu D, Yuan C, Ma C, Jiang B. Preoperative neutrophil-to-lymphocyte ratio and tumor-related factors to predict lymph node metastasis in patients with pancreatic ductal adenocarcinoma (PDAC). Oncotarget. 2016;7(45):74314-24.

16. Pillay J, Tak T, Kamp VM, Koenderman L. Immune suppression by neutrophils and granulocytic myeloid-derived suppressor cells: similarities and differences. Cell Mol Life Sci. 2013;70(20):3813-27.

17. Galdiero MR, Garlanda C, Jaillon S, Marone G, Mantovani A. Tumor associated macrophages and neutrophils in tumor progression. J Cell Physiol. 2013;228(7):1404-12.

18. Fridlender ZG, Albelda SM. Tumor-associated neutrophils: friend or foe? Carcinogenesis. 2012;33(5):949-55.

19. Mishalian I, Bayuh R, Levy L, Zolotarov L, Michaeli J, Fridlender ZG. Tumorassociated neutrophils (TAN) develop pro-tumorigenic properties during tumor progression. Cancer Immunol Immunother. 2013;62(11):1745-56.

20. Sagiv JY, Michaeli J, Assi S, Mishalian I, Kisos H, Levy L, Damti P, Lumbroso D, Polyansky L, Sionov RV, et al. Phenotypic diversity and plasticity in circulating neutrophil subpopulations in cancer. Cell Rep. 2015;10(4):562-73.

21. Zhou SL, Zhou ZJ, Hu ZQ, Huang XW, Wang Z, Chen EB, Fan J, Cao Y, Dai Z, Zhou J. Tumor-associated neutrophils recruit macrophages and T-regulatory cells to promote progression of hepatocellular carcinoma and resistance to Sorafenib. Gastroenterology. 2016;150(7):1646-58. e1617

22. Hattar K, Franz K, Ludwig M, Sibelius U, Wilhelm J, Lohmeyer J, Savai R, Subtil FS, Dahlem G, Eul B, et al. Interactions between neutrophils and nonsmall cell lung cancer cells: enhancement of tumor proliferation and inflammatory mediator synthesis. Cancer Immunol Immunother. 2014;63(12): 1297-306.

23. Galdiero MR, Bianchi P, Grizzi F, Di Caro G, Basso G, Ponzetta A, Bonavita E, Barbagallo M, Tartari S, Polentarutti N, et al. Occurrence and significance of tumor-associated neutrophils in patients with colorectal cancer. Int J Cancer. 2016;139(2):446-56

24. Kim J, Bae JS. Tumor-associated macrophages and neutrophils in tumor microenvironment. Mediat Inflamm. 2016;2016:6058147.

25. Qian Y, Wang Y, Li DS, Zhu YX, Lu ZW, Ji QH, Yang G. The chemokine receptor-CXCR2 plays a critical role in the invasion and metastases of oral squamous cell carcinoma in vitro and in vivo. J Oral Pathol Med. 2014;43(9):658-66.

26. Li L, Xu L, Yan J, Zhen ZJ, Ji Y, Liu CQ, Lau WY, Zheng L, Xu J. CXCR2-CXCL1 axis is correlated with neutrophil infiltration and predicts a poor prognosis in hepatocellular carcinoma. J Exp Clin Cancer Res. 2015;34:129.

27. Sui P, Hu P, Zhang T, Zhang X, Liu Q, Du J. High expression of CXCR-2 correlates with lymph node metastasis and predicts unfavorable prognosis in resected esophageal carcinoma. Med Oncol. 2014;31(2):809.

28. Nishi T, Takeuchi H, Matsuda S, Ogura M, Kawakubo H, Fukuda K, Nakamura R, Takahashi T, Wada N, Saikawa Y, et al. CXCR2 expression and postoperative complications affect long-term survival in patients with esophageal cancer. World J Surg Oncol. 2015;13:232.

29. Jablonska J, Wu CF, Andzinski L, Leschner S, Weiss S. CXCR2-mediated tumor-associated neutrophil recruitment is regulated by IFN-beta. Int J Cancer. 2014;134(6):1346-58.

30. Shibutani M, Maeda K, Nagahara H, Ohtani H, Iseki Y, Ikeya T, Sugano K, Hirakawa K. The prognostic significance of a postoperative systemic 
inflammatory response in patients with colorectal cancer. World J Surg Oncol. 2015;13:194.

31. Fridlender ZG, Sun J, Kim S, Kapoor V, Cheng G, Ling L, Worthen GS, Albelda SM. Polarization of tumor-associated neutrophil phenotype by TGF-beta: "N1" versus "N2" TAN. Cancer Cell. 2009;16(3):183-94.

32. Wang J, Jia Y, Wang N, Zhang $X$, Tan B, Zhang G, Cheng Y. The clinical significance of tumor-infiltrating neutrophils and neutrophil-to-CD8+ lymphocyte ratio in patients with resectable esophageal squamous cell carcinoma. J Transl Med. 2014;12:7.

33. Yoon J, Terada A, Kita H. CD66b regulates adhesion and activation of human eosinophils. J Immunol. 2007;179(12):8454-62.

34. Carus A, Ladekarl M, Hager H, Nedergaard BS, Donskov F. Tumourassociated CD66b+ neutrophil count is an independent prognostic factor for recurrence in localised cervical cancer. Br J Cancer. 2013;108(10):2116-22.

35. Rymaszewski AL, Tate E, Yimbesalu JP, Gelman AE, Jarzembowski JA, Zhang $\mathrm{H}$, Pritchard KA, Vikis HG. The role of neutrophil myeloperoxidase in models of lung tumor development. Cancers. 2014:6(2):1111-27.

36. Ross B. CD32-mediated suppression of plasma cells in patients with multiple myeloma. Haematologica. 2006;91(7):869A.

\section{Ready to submit your research? Choose BMC and benefit from:}

- fast, convenient online submission

- thorough peer review by experienced researchers in your field

- rapid publication on acceptance

- support for research data, including large and complex data types

- gold Open Access which fosters wider collaboration and increased citations

- maximum visibility for your research: over $100 \mathrm{M}$ website views per year

At BMC, research is always in progress.

Learn more biomedcentral.com/submissions 